\title{
Astrocytes are important mediators of $A \beta$-induced neurotoxicity and tau phosphorylation in primary culture
}

\author{
CJ Garwood ${ }^{1}$, AM Pooler ${ }^{1}$, J Atherton ${ }^{1}$, DP Hanger ${ }^{1}$ and W Noble ${ }^{\star, 1}$
}

\begin{abstract}
Alzheimer's disease (AD) is pathologically characterised by the age-dependent deposition of $\beta$-amyloid $(A \beta)$ in senile plaques, intraneuronal accumulation of tau as neurofibrillary tangles, synaptic dysfunction and neuronal death. Neuroinflammation, typified by the accumulation of activated microglia and reactive astrocytes, is believed to modulate the development and/or progression of $A D$. We have used primary rat neuronal, astrocytic and mixed cortical cultures to investigate the contribution of astrocyte-mediated inflammatory responses during $A \beta$-induced neuronal loss. We report that the presence of small numbers of astrocytes exacerbate $A \beta$-induced neuronal death, caspase-3 activation and the production of caspase-3-cleaved tau. Furthermore, we show that astrocytes are essential for the $A \beta$-induced tau phosphorylation observed in primary neurons. The release of soluble inflammatory factor(s) from astrocytes accompanies these events, and inhibition of astrocyte activation with the anti-inflammatory agent, minocycline, reduces astrocytic inflammatory responses and the associated neuronal loss. $A \beta$-induced increases in caspase-3 activation and the production of caspase-3-truncated tau species in neurons were reduced when the astrocytic response was attenuated with minocycline. Taken together, these results show that astrocytes are important mediators of the neurotoxic events downstream of elevated $A \beta$ in models of $A D$, and suggest that mechanisms underlying pro-inflammatory cytokine release might be an important target for therapy.
\end{abstract}

Cell Death and Disease (2011) 2, e167; doi:10.1038/cddis.2011.50; published online 2 June 2011

Subject Category: Neuroscience

Alzheimer's disease (AD) is a neurodegenerative disorder pathologically characterised by deposits of $\beta$-amyloid $(A \beta)$ in senile plaques, intracellular neurofibrillary tangles comprising hyperphosphorylated tau, synaptic dysfunction and neuronal death, some or all of which are believed to cause the cognitive and behavioural deficits that typify this disease. It is now widely recognised that neuroinflammation is a prominent feature of $A D$ brain, with inflammatory responses playing a significant role in modulating disease progression. ${ }^{1}$

Prolonged and widespread activation of microglia and astrocytes are apparent in AD brain, in which the severity of glial activation correlates with the extent of brain atrophy ${ }^{2}$ and cognitive decline. ${ }^{3}$ The majority of transgenic rodent models of $A D$ also exhibit substantial reactive gliosis and accumulation of activated astrocytes in affected brain regions. ${ }^{4,5}$ These features are often observed before the appearance of plaque and tangle pathology, ${ }^{6,7}$ and the degree of inflammatory cell activation correlates with pathology development and neuronal death in mouse models of $A D$. $^{6,8}$

The role of microglia in the development and/or progression of $A D$ is somewhat controversial. Phagocytosis of $A \beta$ by microglia is believed to be a protective mechanism, ${ }^{9}$ at least in younger animals; ${ }^{10}$ however, neuronal loss also results from increased pro-inflammatory cytokine production by microglia in models of $A D .^{10}$ In addition to the influence of microglia, an important role of astrocytes in regulating $A \beta$-induced neuronal death has recently been illustrated. ${ }^{11}$

Both astrocytes and microglia release a myriad of pro- and anti- inflammatory cytokines, including interleukins (ILs), interferons (IFNs) and tumour necrosis factors (TNFs), as well as chemokines, a family of small pro-inflammatory cytokines that includes macrophage inflammatory proteins and monocyte chemoattractant proteins. ${ }^{12}$ Receptor binding of cytokines stimulates a variety of intracellular signalling pathways that have been implicated in $A D$, including the activation of protein kinase C, c-Jun N-terminal kinase (JNK), p38 mitogen-activated protein kinase (p38/MAPK), PI3 kinase, extracellular signalling-related kinase (ERK), as well as activation of caspase- 1 and $-3 .^{13,14}$

Caspases are closely associated with induction of the apoptotic cascade, and activation of caspase-3 is both necessary and sufficient to trigger apoptotic cell death in response to elevated $\mathrm{A} \beta$ in $\mathrm{AD} .^{15}$ In addition, $\mathrm{A} \beta$-induced caspase- 3 activation causes abnormal processing of the microtubule-associated protein tau in models of $A D .^{16,17}$ Cleavage of tau by caspase-3 at Asp421 generates a

\footnotetext{
${ }^{1}$ Department of Neuroscience, King's College London, MRC Centre for Neurodegeneration Research, Institute of Psychiatry, London, UK

*Corresponding author: W Noble, Department of Neuroscience (PO37), King's College London, MRC Centre for Neurodegeneration Research, Institute of Psychiatry, De Crespigny Park, London SE5 8AF, UK. Tel: + 440207848 0578; Fax: + 440207708 0017; E-mail: Wendy.Noble@kcl.ac.uk

Keywords: Alzheimer's disease; astrocytes; $\beta$-amyloid; minocycline; tau; inflammation

Abbreviations: $\mathrm{AD}$, Alzheimer's disease; $\mathrm{A} \beta, \beta$-amyloid; CINC, cytokine-induced neutrophil chemoattractant; DIV, days in vitro; ERK, extracellular signalling-related kinase; GFAP, glial fibrillary acidic protein; IFN, interferon; IL, interleukin; JNK, c-Jun N-terminal kinase; LDH, lactate dehydrogenase; MAPK, mitogen-activated protein kinase; MIG, monokine induced by IFN- $\gamma$; NO, nitric oxide; SDS, sodium dodecyl sulphate; TNF, tumour necrosis factor

Received 20.12.10; revised 11.4.11; accepted 03.5.11; Edited by A Verkhratsky
} 
truncated form that aggregates more rapidly, and to a greater extent, than full-length tau, ${ }^{18,19}$ and caspase-cleaved tau fragments directly reduce neuronal viability. ${ }^{16}$ Aberrant tau phosphorylation in response to elevated $A \beta$ also contributes to tau aggregation and fibrillisation in $\mathrm{AD} .{ }^{20}$ Putative tau kinases that phosphorylate tau in response to $A \beta$ include cyclindependent kinase-5, glycogen synthase kinase-3 $\beta$, p38/ MAPK, JNK-1 and ERK, ${ }^{21,22}$ several of which are activated by increased production or release of cytokines. ${ }^{13}$

We have shown previously that the neuroprotective agent, minocycline, reduces tau phosphorylation and proteolysis in both $\mathrm{A} \beta$-treated primary cortical cultures and also in htau mice, which progressively accumulate hyperphosphorylated aggregated tau and show significant neuronal loss in the cortex and hippocampus in the absence of elevated $\mathrm{A} \beta{ }^{17}$ In htau mice, the impact of minocycline on tau pathology was not associated with tau kinase inhibition, ${ }^{17}$ but correlated with reductions in astrocytosis and in the CNS burden of proinflammatory factors. ${ }^{6}$ Indeed, the cortical amounts of individual inflammatory cytokines correlated strongly with phosphorylated tau load. ${ }^{6}$ Here, we have investigated the role of astrocytes in $\mathrm{A} \beta$-induced tau phosphorylation and cleavage, as well as determining the influence of astrocytes in mediating $\mathrm{A} \beta$-induced neuronal death.

\section{Results}

Astrocytes accelerate $\mathbf{A} \boldsymbol{\beta}$-induced neurotoxicity. We first compared primary cortical cultures in which neurons were either cultured alone (neuronal cultures) or were in co-culture with astrocytes (mixed cultures). Immunolabelling with celltype-specific antibodies identified constituent cells in the cultures and revealed an absence of glia in neuronal cultures. Mixed cultures were predominantly neuronal, containing $4.4 \pm 0.4 \%$ astrocytes and a negligible amount of microglia $(<0.01 \%)$ after 7 days in vitro (DIV).

To determine whether or not astrocytes influence $\mathrm{A} \beta$-induced neurotoxicity, mixed and neuronal cultures were treated with $10 \mu \mathrm{M}$ soluble oligomeric $\mathrm{A} \beta$ and lactate dehydrogenase $(\mathrm{LDH})$ release was used to measure cell viability. $\mathrm{A} \beta$ treatment of mixed cultures, but not neuronal cultures, resulted in a statistically significant increase in LDH release at both 48 and $72 \mathrm{~h}$ (Figures $1 \mathrm{a}$ and b; $P<0.001$ ). In contrast, significantly increased $\mathrm{LDH}$ release was only observed in neuronal cultures following a $72 \mathrm{~h}$ treatment with $\mathrm{A} \beta$ (Figure 1b; $P<0.01$ ). $\mathrm{A} \beta$ treatment did not cause increased release of $\mathrm{LDH}$ from cultured astrocytes (Figure 1c), indicating that the presence of astrocytes significantly accelerates $\mathrm{A} \beta$-induced neuronal death. a
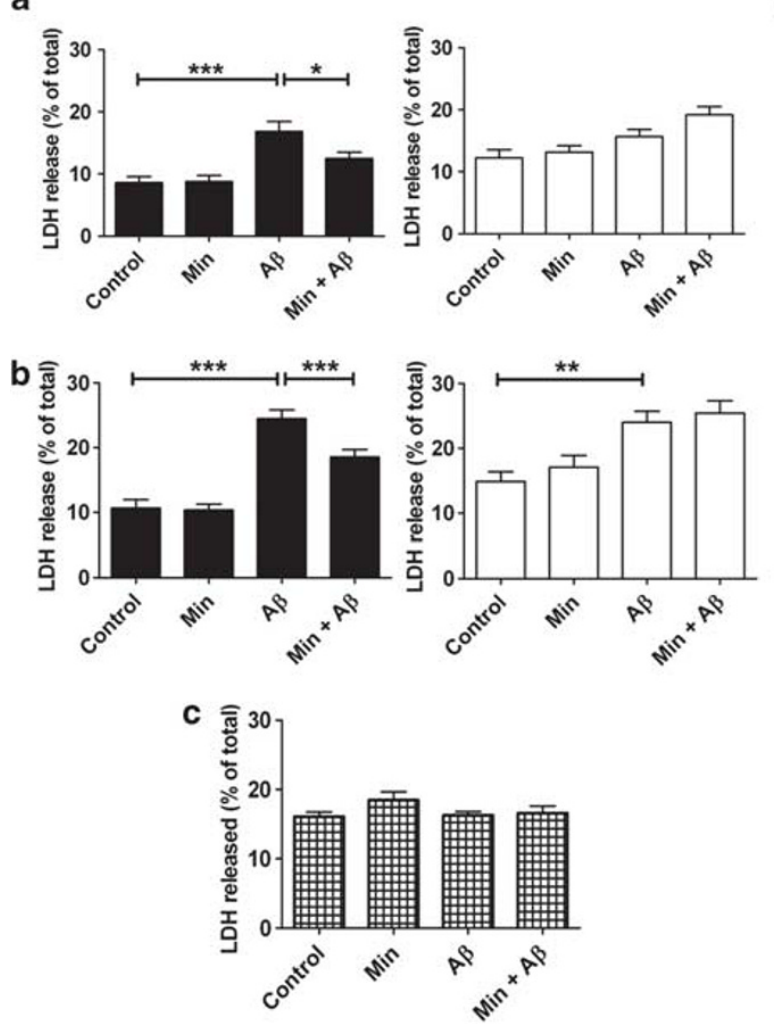

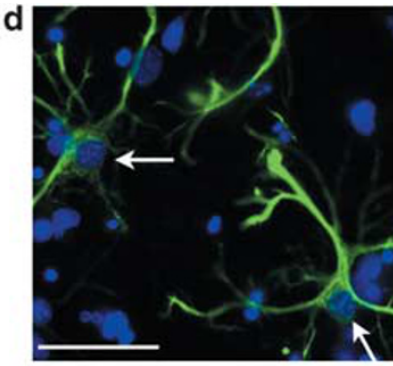

GFAP

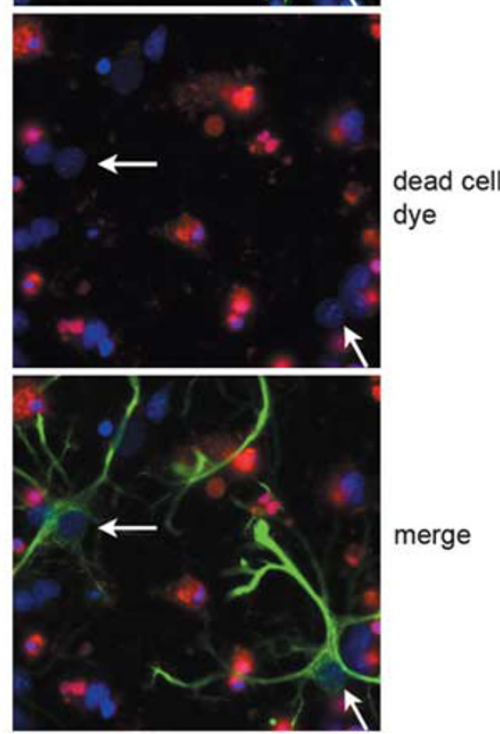

Figure 1 Astrocytes accelerate $\beta$-amyloid (A $\beta$ )-induced neurotoxicity. Bar charts show LDH release from rat primary mixed (black bars) and neuronal (white bars) cultures exposed to $10 \mu \mathrm{M} \mathrm{A} \beta$ for (a) 48 or (b) $72 \mathrm{~h}$, with and without a $24 \mathrm{~h}$ pretreatment with $20 \mu \mathrm{M}$ minocycline (Min). (c) LDH release from rat primary astrocyte cultures exposed to $10 \mu \mathrm{M} \mathrm{A} \beta$ for $72 \mathrm{~h}$, with and without a $24 \mathrm{~h}$ pretreatment with $20 \mu \mathrm{M}$ Min. Values represent LDH release from cells into the medium as a percentage of total LDH, shown as mean \pm S.E.M. Experiments were performed in triplicate $(N=9-15)$. ${ }^{\star} P<0.05 ;{ }^{\star \star} P<0.01 ;{ }^{\star \star \star} P<0.001$. (d) Representative images from mixed cortical cultures treated for $48 \mathrm{~h}$ with $\mathrm{A} \beta$, labelled with a dead cell dye (red), and then fixed and immunostained with an antibody against glial fibrillary acidic protein (GFAP) (green). Bisbenzimide was used to stain nuclei (blue). The red dye labels dead cells. Arrows indicate astrocytes that are unaffected by A $\beta$. Scale bar: $50 \mu \mathrm{m}, N=3$ 

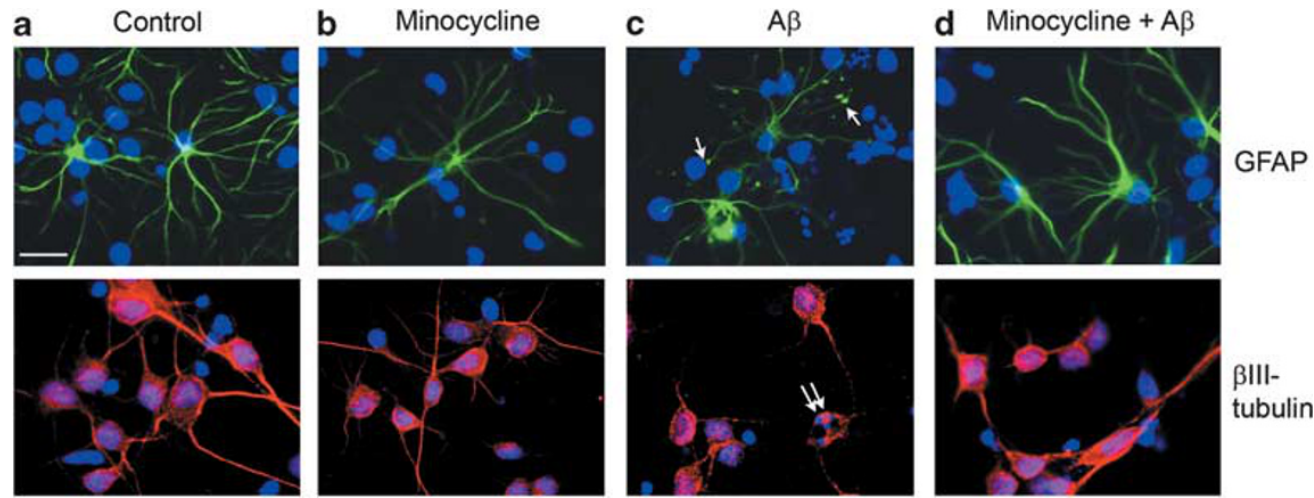

Figure $2 \beta$-Amyloid $(\mathrm{A} \beta)$ induces morphological changes in astrocytes. Representative images from mixed cortical cultures treated for $48 \mathrm{~h}$ with $(\mathbf{a})$ vehicle, $(\mathrm{b}) 20 \mu \mathrm{M}$ minocycline, (c) $10 \mu \mathrm{M} \mathrm{A} \beta$ or (d) $10 \mu \mathrm{M} \mathrm{A} \beta$ with a $24 \mathrm{~h}$ pretreatment with $20 \mu \mathrm{M}$ minocycline. Cells were fixed and immunostained with antibodies against glial fibrillary acidic protein (GFAP) (green), to label astrocytes, or $\beta$ III-tubulin (red), to label neurons. Bisbenzimide was used to stain nuclei (blue). Single arrows indicate astrocytic terminal swellings, and double arrows indicate apoptotic nuclei. Scale bar: $50 \mu \mathrm{m} . N=9$

To confirm that neurons, and not astrocytes, are susceptible to $\mathrm{A} \beta$ toxicity in mixed culture, a fixable dead cell staining kit was used in conjunction with immunolabelling of astrocytes (Figure 1d). As expected, this method revealed a proportion of dead cells following $\mathrm{A} \beta$ treatment. However, glial fibrillary acidic protein (GFAP)-immunoreactive astrocytes were not labelled by the dead cell dye. Furthermore, the nuclei of astrocytes were not fragmented or condensed, unlike many of the surrounding neurons (Figure 2d, white arrows). In further support of this finding, no alteration in the abundance of astrocytes was apparent in mixed cultures following $\mathrm{A} \beta$ treatment (Supplementary Figure 1). These results support those from the LDH assays and conclusively show that in mixed cell cultures, only neurons are susceptible to $\mathrm{A} \beta$-induced toxicity.

To determine if astrocytic inflammatory responses are involved in the acceleration of $\mathrm{A} \beta$-induced neurotoxicity, cultures were pretreated with the anti-inflammatory agent minocycline. LDH release was not significantly affected by minocycline alone in any of the cultures. Pretreatment of mixed, but not neuronal cultures with minocycline resulted in reduced $\mathrm{LDH}$ release from cultures treated with $\mathrm{A} \beta$ for 48 or $72 \mathrm{~h}$ (Figures $1 \mathrm{a}$ and b; $P<0.05$ and $P<0.001$, respectively). This finding suggests that minocycline may reduce $\mathrm{A} \beta$-induced neuronal death by suppressing the inflammatory response of astrocytes.

$\mathbf{A} \boldsymbol{\beta}$ induces morphological changes in astrocytes. Astrocytes are highly dynamic cells that exhibit morphological changes depending on their cellular environment. ${ }^{23,24}$ In primary culture, the morphology of astrocytes is considered a reliable marker of their activation state. ${ }^{25}$ Therefore, we next examined astrocyte morphology in response to $\mathrm{A} \beta$ and minocycline in mixed cultures. Under control conditions, and following treatment with minocycline alone, astrocytes appeared as stellate process-bearing cells (Figures $2 \mathrm{a}$ and $\mathrm{b}$ ), in line with previous reports. ${ }^{24}$ Following exposure to $\mathrm{A} \beta$, there were clear and robust alterations in astrocyte morphology with the appearance of convoluted processes and terminal swellings (Figure 2c, single arrows). Such changes are believed to represent characteristics of astrocyte activation in culture. ${ }^{25,26}$ These alterations are not indicative of dystrophic astrocytes as $\mathrm{A} \beta$-treated astrocytes do not label with the dead cell dye (Figure 1d). Pretreatment with minocycline before $\mathrm{A} \beta$ prevented these $\mathrm{A} \beta$-induced morphological changes in mixed cultures (Figure 2d).

In contrast, following $\mathrm{A} \beta$ treatment, neurons displayed condensed and fragmented neuronal nuclei (double arrows, Figure 2c) and a disrupted tubulin cytoskeleton (Figure 2c). These results add further support to our finding that neurons, rather than astrocytes, are susceptible to $\mathrm{A} \beta$ toxicity in mixed culture. Pretreatment of mixed cultures with minocycline before the addition of $\mathrm{A} \beta$ reduced these markers of neuronal death (Figure 2d).

These results suggest that astrocytes are activated in response to $\mathrm{A} \beta$, which is an event closely associated with $\mathrm{A} \beta$-induced neuronal death. Furthermore, minocyclinemediated neuroprotection from $\mathrm{A} \beta$ toxicity appears to be associated with the inhibition of astrocyte activation.

\section{A soluble factor secreted from astrocytes mediates} A $\beta$-induced neuronal death. Astrocytes may mediate neuronal responses both by direct membrane interactions ${ }^{27}$ and by the release of factors that bind to neuronal receptors. ${ }^{11}$ To investigate the mechanism underlying the astrocyte-mediated acceleration of $\mathrm{A} \beta$-induced neuronal death, we treated primary cortical astrocyte-enriched cultures with $\mathrm{A} \beta$ for $48 \mathrm{~h}$, a treatment that is not toxic to these cells (Figure 1c). Addition of conditioned medium from $\mathrm{A} \beta$-treated astrocytes to cultured neurons resulted in increased $\mathrm{LDH}$ release (Figure $3 \mathrm{a}$ ). As a control, $\mathrm{A} \beta$ was incubated in astrocyte culture medium, in the absence of cells, for $48 \mathrm{~h}$, and application of this medium to neurons did not affect cell viability (Figure $3 b$ ). These results show that a component of the astrocyte-conditioned medium mediates neuronal death. ELISA measurement of $A \beta$ in conditioned medium revealed that the amount of $A \beta$ reduced from 10 to $1.08 \pm 0.06 \mu \mathrm{M}$ after $48 \mathrm{~h}$ incubation with primary astrocyte cultures. As $1 \mu \mathrm{M} \mathrm{A} \beta$ is insufficient to induce neuronal death in these cultures, we conclude that a soluble factor released from astrocytes is important for $\mathrm{A} \beta$-induced neurotoxicity. 

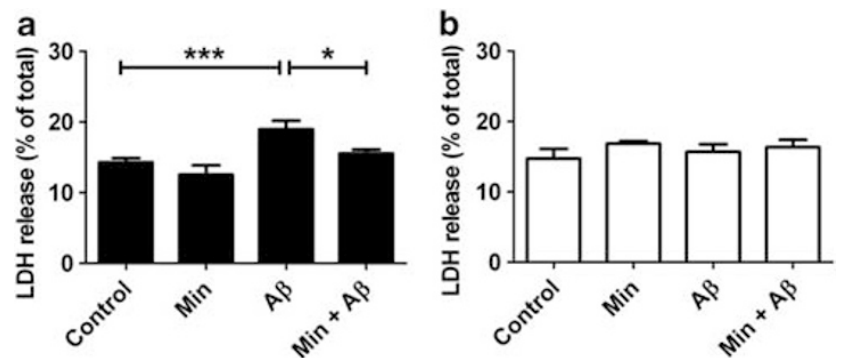

Figure 3 A soluble factor secreted from astrocytes mediates $\beta$-amyloid $(\mathrm{A} \beta)$ induced neuronal death. Bar charts show lactate dehydrogenase (LDH) release into culture medium from rat primary neuronal cultures exposed to (a) conditioned medium from primary astrocyte cultures treated with $10 \mu \mathrm{M} \mathrm{A} \beta$ and/or $20 \mu \mathrm{M} \mathrm{Min}$, and (b) cell-free primary astrocyte culture medium infused with $10 \mu \mathrm{M}$ Ab for $48 \mathrm{~h}$, with and without the addition of $20 \mu \mathrm{M}$ minocycline (Min). Values are LDH release from cells into medium expressed as a percentage of total LDH, shown as mean \pm S.E.M. Experiments were performed in triplicate $(N=9)$. ${ }^{*} P<0.05$; ${ }^{\star * *} P<0.001$

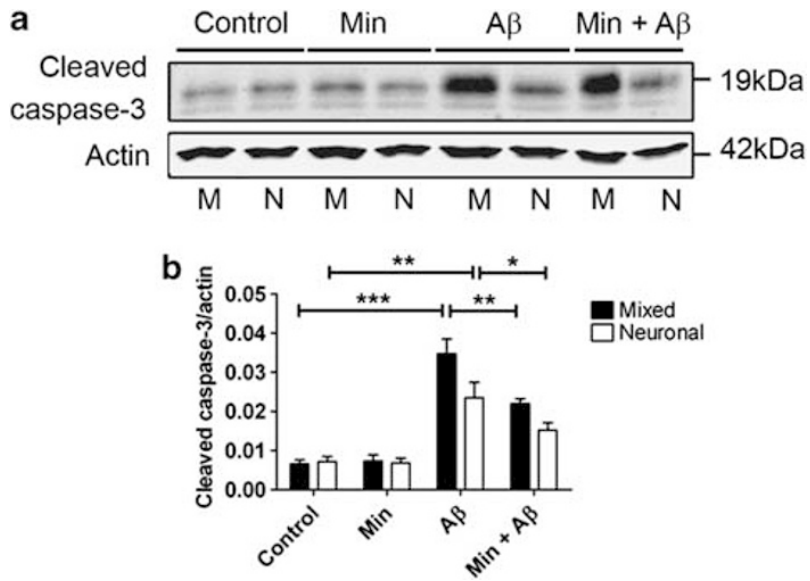

Figure 4 Astrocytes increase $\beta$-amyloid $(\mathrm{A} \beta$ )-induced caspase-3 activity. Western blots of cell lysates from rat primary mixed $(\mathrm{M})$ and neuronal $(\mathrm{N})$ cultures. Cultures were exposed to $10 \mu \mathrm{M} \mathrm{A} \beta$ for $48 \mathrm{~h}$ with and without pretreatment with $20 \mu \mathrm{M}$ minocycline for $24 \mathrm{~h}$. (a) Representative western blots probed with an antibody against cleaved caspase-3 (17 and $19 \mathrm{kDa})$. Blots were also probed with $\beta$-actin (42 kDa) as a loading control. Molecular weight markers are indicated $(\mathrm{kDa})$. (b) Bar chart showing the amount of cleaved caspase-3 following standardisation to $\beta$-actin in each sample. Data are mean \pm S.E.M. Experiments were performed in triplicate $(N=12) .{ }^{*} P<0.05 ;{ }^{* \star} P<0.01 ;{ }^{* * *} P<0.001$

Pretreatment of astrocyte-enriched cultures with minocycline before $\mathrm{A} \beta$ application significantly reduced neuronal death in this model system (Figure $3 b$ ), suggesting that the neurotoxic astrocyte-derived soluble factor(s) released in response to $\mathrm{A} \beta$ may be an inflammatory mediator.

Astrocytes increase $\mathbf{A} \boldsymbol{\beta}$-induced caspase-3 activity. The finding that astrocytes accelerate $\mathrm{A} \beta$-induced neuronal toxicity led us to investigate whether caspase-3 activity was influenced by astrocytes, as increased caspase- 3 activity is associated with $A \beta$-induced neurotoxicity. ${ }^{15-17}$ Furthermore, we have shown that minocycline inhibits caspase-3 activation in both primary cortical cultures and in htau mice, ${ }^{17}$ the former closely associated with protection from $\mathrm{A} \beta$-induced neurotoxicity. To investigate if astrocytes influence caspase-3 activity, we analysed cell lysates from $\mathrm{A} \beta$ - and/or minocycline-treated mixed and neuronal cultures on western blots (Figure 4).

Caspase- 3 is activated by cleavage, generating fragments of approximately 17 and $19 \mathrm{kDa}$ from the full-length (35 kDa) protein. We therefore measured caspase-3 activity using an antibody against cleaved caspase-3 (Figure 4a). Treatment of both mixed and neuronal cultures with $A \beta$ for $48 \mathrm{~h}$ significantly increased the amount of cleaved caspase- 3 present in cell lysates, when compared with those from vehicle-treated cells ( $P<0.001$ and $P<0.01$, respectively). The activation of caspase- 3 by $A \beta$ was significantly enhanced in mixed cultures when compared with neuronal cultures $(P<0.05)$, indicating that astrocytes exacerbate $A \beta$-induced caspase- 3 activation, and this is likely related to the impact of astrocytes in mediating $\mathrm{A} \beta$-induced neuronal death. Pretreatment of both mixed and neuronal cultures with minocycline reduced the $\mathrm{A} \beta$-induced accumulation of cleaved caspase-3 (Figure $4 \mathrm{~b}$; $P<0.01$ and $P<0.05$, respectively). This suggests that while astrocytic inflammatory responses may exacerbate caspase3 activation in response to $\mathrm{A} \beta$, both neurons and astrocytes are targeted by minocycline to reduce caspase- 3 activation.

Astrocytes exacerbate $\mathbf{A} \boldsymbol{\beta}$-mediated tau truncation. The truncation of tau by caspase- 3 is believed to be an important event during the development of tau pathology in $A D$, with caspase-3-cleaved tau more prone to aggregation than fulllength tau. ${ }^{18,19} \mathrm{~A} \beta$ has previously been shown to induce the generation of caspase-3-cleaved tau species in primary cortical cultures, ${ }^{17}$ an event associated with neuronal death. ${ }^{19,28}$

Therefore, cell lysates from mixed and neuronal cultures were assessed for caspase-cleaved tau by western blotting using an antibody that specifically detects tau that has been cleaved at Asp421 (Figures 5a and b). In both types of control cultures, small amounts of caspase-3-cleaved tau were detected at approximately $50 \mathrm{kDa}$, in line with our previous findings. ${ }^{17} \mathrm{~A} \beta$ treatment significantly increased the amount of cleaved tau present in both mixed and neuronal cultures (Figure 5b; $P<0.001$ and $P<0.01$, respectively); however, significantly more caspase-3-cleaved tau was apparent following $\mathrm{A} \beta$ treatment of mixed cultures when compared with neuronal cultures $(P<0.05)$. Cultured astrocytes contain only trace amounts of tau (Figure $5 \mathrm{c}$ ), suggesting that the tau measured in the mixed cultured is of neuronal origin, and that the presence of astrocytes significantly potentiates the generation of caspase-cleaved tau in neurons in response to $\mathrm{A} \beta$ (Figures $5 \mathrm{a}$ and $\mathrm{b}$ ). Pretreatment of mixed, but not neuronal, cultures with minocycline significantly reduced the amount of caspase-3-cleaved tau species generated in response to $\mathrm{A} \beta$ treatment $(P<0.01)$, again suggesting that astrocytic inflammatory responses may have a role in the $\mathrm{A} \beta$-induced cell signalling that leads to caspase activation and tau cleavage.

Astrocytes are necessary for A $\beta$-induced tau phosphorylation. Truncation of tau is closely associated 

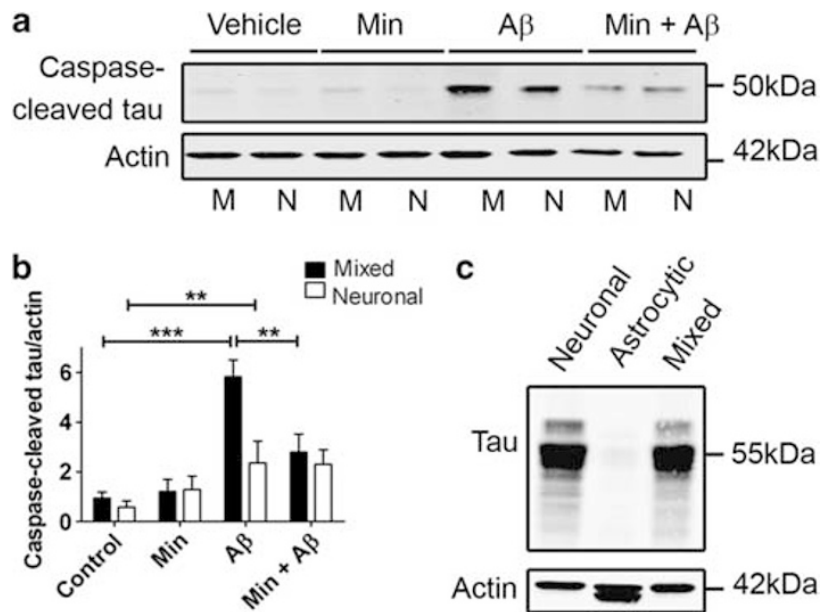

Figure 5 Astrocytes exacerbate $\beta$-amyloid $(\mathrm{A} \beta)$-induced tau truncation by caspase-3. (a) Western blots of cell lysates from rat primary mixed (M) and neuronal (N) cultures. Cultures were exposed to $10 \mu \mathrm{M} \mathrm{A} \beta$ for $48 \mathrm{~h}$ with and without pretreatment with $20 \mu \mathrm{M}$ minocycline for $24 \mathrm{~h}$. Representative western blots probed with an antibody against caspase-3-cleaved tau $(50 \mathrm{kDa})$ are shown. Blots were also probed with $\beta$-actin $(42 \mathrm{kDa})$ as a loading control. Molecular weight markers are indicated (kDa). (b) Bar chart showing the amount of caspase-3-cleaved tau as a proportion of $\beta$-actin in each sample. (c) Representative western blots of cell lysates from primary neuron, astrocyte and mixed cultures, probed with antibodies against total tau $(55 \mathrm{kDa})$ and $\beta$-actin $(42 \mathrm{kDa})$. Data are mean $\pm \mathrm{S}$.E.M. Experiments were performed in triplicate $(N=12) .{ }^{*} P<0.05 ;{ }^{* *} P<0.01 ;{ }^{* \star} P<0.001$

with its phosphorylation, ${ }^{29}$ and $\mathrm{A} \beta$-induced tau phosphorylation is widely regarded as an important event in $A D{ }^{22}$ To determine if astrocytes mediate $\mathrm{A} \beta$-induced tau phosphorylation in our model system, cell lysates from $\mathrm{A} \beta$-treated mixed and neuronal cultures were assessed by western blotting. Blots were probed with antibodies against total tau, and tau phosphorylated at serine (Ser) 396/404 (PHF1) and Ser202 (CP13), sites previously shown to be hyperphosphorylated in AD brain. ${ }^{30}$

A $\beta$ treatment of mixed cultures increased tau phosphorylation at both Ser396/404 $(P<0.01)$ and Ser202 $(P<0.001)$ (Figures $6 \mathrm{a}$ and $\mathrm{b}$ ), when compared with vehicle-treated cultures. In addition to full-length tau, an additional tau species of approximately $50 \mathrm{kDa}$ was labelled with both phosphorylation-specific tau antibodies on blots (Figure 6a). Co-labelling of blots with antibodies against phosphorylated Ser396 on tau and caspase-3-cleaved tau revealed that the $50 \mathrm{kDa}$ band corresponds to caspase-3-truncated tau (Figure 6c). This suggests that the generation of caspase-3-cleaved tau in response to elevated $\mathrm{A} \beta$ occurs either before or in concert with tau phosphorylation, at least that found at the sites examined here. No increase in tau phosphorylation at either of these epitopes was observed upon $A \beta$ treatment of neuronal cultures. This finding shows that astrocytes are necessary for $\mathrm{A} \beta$-induced tau phosphorylation, at least in this model system. Pretreatment of mixed cultures with minocycline significantly reduced tau phosphorylation at Ser396/404 and Ser202 $(P<0.05$ for both). Thus, astrocytic inflammatory responses likely have a role in $A \beta$-induced tau phosphorylation. Furthermore, both astrocyte-mediated tau phosphorylation and the generation of caspase-cleaved tau are closely associated with $A \beta$-induced neurotoxicity.
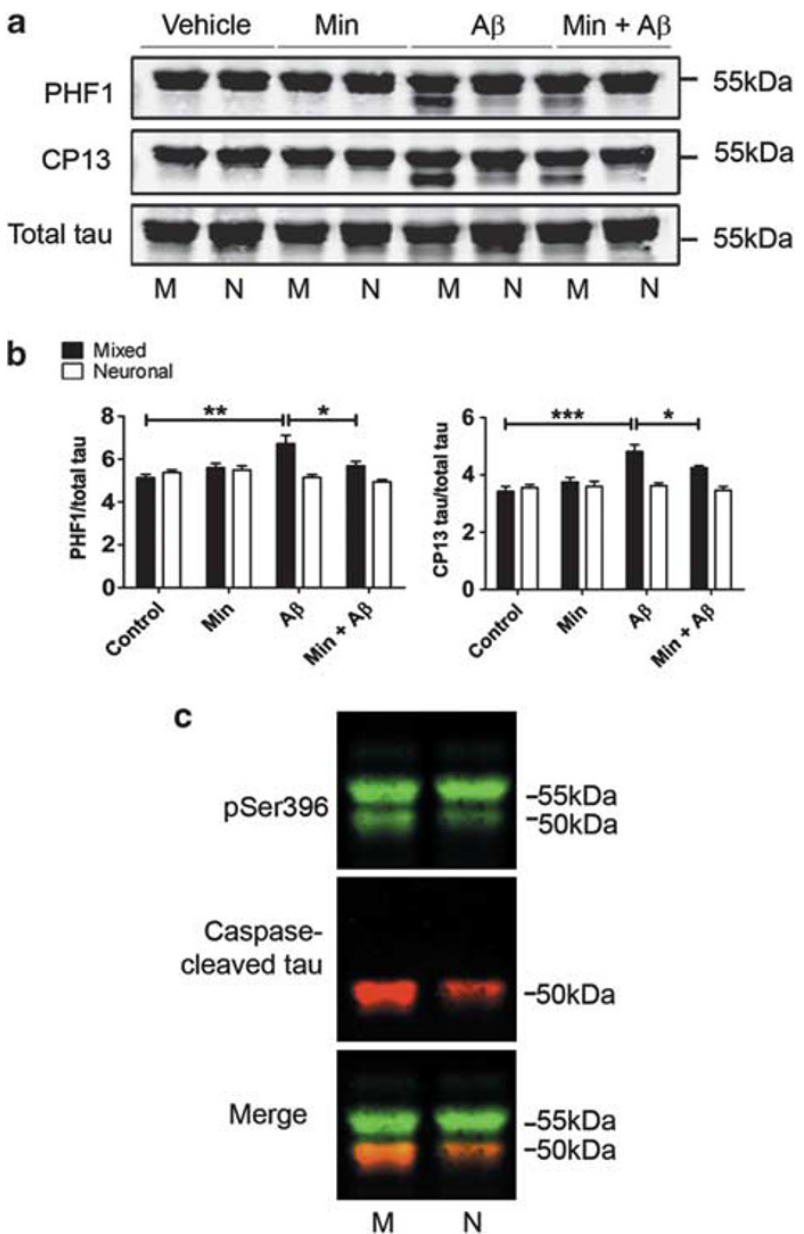

Figure 6 Astrocytes are necessary for $\beta$-amyloid $(\mathrm{A} \beta)$-induced tau phosphorylation. Western blots of cell lysates from rat primary mixed $(\mathrm{M})$ and neuronal $(\mathrm{N})$ cultures treated with $10 \mu \mathrm{M} \mathrm{A} \beta$ for $48 \mathrm{~h} \pm 20 \mu \mathrm{M}$ minocycline (Min). (a) Representative western blots probed with antibodies recognising tau phosphorylated at Ser396/404 (PHF1) and Ser202 (CP13), and total tau. (b) Bar chart shows the amount of tau phosphorylated at either Ser396/404 (PHF1) or Ser202 (CP13) as a proportion of total tau. (c) Representative western blots of cell lysates from $\mathrm{A} \beta$-treated mixed cultures and neuronal cultures, probed with antibodies recognising tau phosphorylated at Ser396 (green) and caspase-cleaved tau (red). Molecular weight markers are indicated $(\mathrm{kDa})$. Data are mean \pm S.E.M. Experiments were performed in triplicate $(N=12) .{ }^{*} P<0.05 ; \quad{ }^{*} P<0.01$; ${ }^{\star \star \star} P<0.001$

The increased release of inflammatory mediators from astrocytes is associated with $A \beta$-induced neurotoxicity. We and others have previously shown that the amounts of specific inflammatory cytokines correlate strongly with pathology development in transgenic mouse models of $A D .^{6,8}$ Therefore, the release of inflammatory mediators from $\mathrm{A} \beta$ - and minocycline-treated astrocyteenriched cultures was measured using cytokine arrays. These arrays were chosen as they allow the unbiased and simultaneous measurement of 29 rat cytokines.

$\mathrm{A} \beta$ treatment of astrocyte-enriched cultures had a significant overall effect on the release of inflammatory cytokines (Figure 3a; $P<0.01$ ), appearing to elevate the medium amounts of the majority of cytokines studied, including 

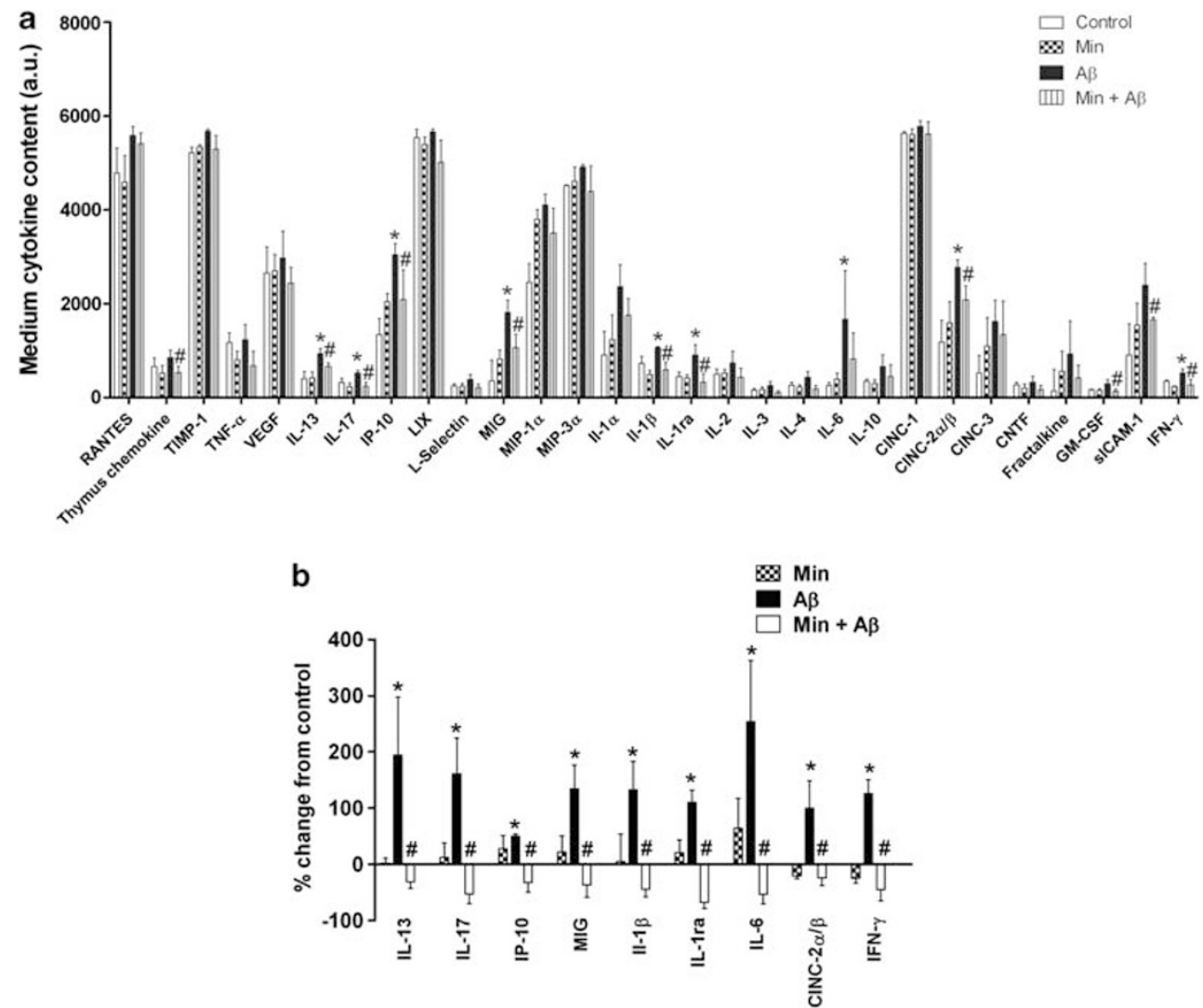

Figure $7 \quad \beta$-Amyloid $(\mathrm{A} \beta)$ treatment increases the release of several inflammatory mediators from astrocytes. Astrocyte cultures were treated with $10 \mu \mathrm{M} \mathrm{A} \beta$ for $48 \mathrm{~h}$ with and without pretreatment with $20 \mu \mathrm{M}$ minocycline (Min). Cytokine arrays were used to detect the amounts of 29 inflammatory mediators in the medium of treated cells. (a) Bar chart shows quantification of medium cytokine amounts in arbitrary densitometry units (a.u.). (b) Bar chart shows percentage change in the media amounts of those cytokines significantly influenced by $A \beta$ and/or minocycline as percentage change from control. Values represent mean \pm S.E.M., $n=3$. ${ }^{*} P<0.05$ when compared with control; ${ }^{\#} P<0.05$ compared with $A \beta$ treated

IL-1 $\beta$, IL-6 and IFN- $\gamma$, each of which has been reported to be increased in response to $\mathrm{A} \beta .{ }^{11}$ Moreover, additional cytokines, not previously identified, were elevated by $A \beta$, including IL-13, IL-17 and IP-10. Statistical analysis revealed that cytokine-induced neutrophil chemoattractant (CINC) $2 \alpha / \beta$, IFN- $\gamma$, IL-1 $\beta$, IL-1ra, IL-6, IL-13, IL-17, IP-10 and monokine induced by IFN- $\gamma$ (MIG) were significantly increased in response to $\mathrm{A} \beta$ treatment (Figures $7 \mathrm{a}$ and $\mathrm{b} ; P<0.05$ for all). Minocycline pretreatment had a significant overall influence on the $A \beta$-induced increases in cytokine media amounts (Figure $3 \mathrm{a} ; P<0.001$ ), with the $A \beta$-induced increases in the nine aforementioned cytokines being significantly reduced by minocycline pretreatment (Figures $7 a$ and $b$; $P<0.05$ for all). These findings indicate a close association between cytokine release and the neurotoxic events that occur downstream of $A \beta$, and suggest that suppression of these inflammatory responses may represent a valid therapeutic strategy for the treatment of $A D$ and related neurodegenerative disorders.

\section{Discussion}

Here we have used cultured neurons and astrocytes to show that astrocytes have an important role in neurodegen- erative events occurring downstream of $A \beta$. We found that astrocytes exacerbate $A \beta$-induced neurotoxicity, caspase- 3 activation and the generation of caspase-3-cleaved tau fragments, and are necessary for $A \beta$-induced tau phosphorylation. A soluble factor secreted from astrocytes appears to have an important role in these processes, and we have identified a panel of inflammatory mediators as candidates whose secretion is increased in response to $\mathrm{A} \beta$. Inhibiting astrocytic inflammatory responses with minocycline reduced or abolished these effects of $A \beta$, suggesting that the soluble factor that has an important role in $\mathrm{A} \beta$-induced toxicity and abnormal tau processing may be an inflammatory cytokine.

Increased LDH release was observed $48 \mathrm{~h}$ after $\mathrm{A} \beta$ treatment in mixed, but not neuronal cultures, suggesting that astrocytes accelerate the neurotoxic effects of $A \beta$. These findings are in agreement with those of Paradisi et al., ${ }^{31}$ who previously reported that $A \beta$-treated astrocytes have deleterious effects on neuronal cultures when co-cultured with neurons. ${ }^{31}$ In addition, we found that the morphology of astrocytes was altered upon treatment with $A \beta$, with astrocytes developing convoluted processes and terminal swellings. Specific labelling of dead cells showed that these morphological alterations are not indicative of atrophic 
cells. Indeed, previous findings indicate that this altered morphological state occurs when astrocytes enter an active, cytokine-secreting state. ${ }^{26}$ Furthermore, similar alterations to astrocytic morphology have been reported in $3 \times \mathrm{Tg}-\mathrm{AD}$ mice in which astrocytes located in close proximity to senile plaques were shown to be hypertrophic, rather than atrophic or dystrophic. ${ }^{32}$

We found that $A \beta$-induced neurotoxicity was associated with increased activity of caspase-3, and that this was exacerbated in the presence of astrocytes. Activated forms of caspase- 3 are found in AD brain, ${ }^{28}$ and we have previously shown that activation of caspase- 3 precedes $A \beta$-induced neuronal death in primary cortical cultures. ${ }^{17}$ The mechanism underlying astrocyte-mediated increased caspase-3 activation in response to $\mathrm{A} \beta$ might, therefore, be attributed to factors that are secreted from astrocytes, or direct neuron-astrocyte interactions. Previous evidence suggests that soluble factors released by astrocytes bind to neuronal plasma membrane receptors inducing various cell signalling pathways. For example, when secreted from astrocytes, TNF- $\alpha$ binds to neuronal $\mathrm{Cd} 120 \mathrm{a} / \mathrm{b}$ receptors triggering caspase activation through death effector domains. ${ }^{33}$

Astrocytes could potentially influence $A \beta$-induced neurotoxicity through direct membrane-associated interactions, as described previously. ${ }^{27}$ However, by treating neurons with the medium from $\mathrm{A} \beta$-treated astrocyte cultures, we established that a soluble factor(s) secreted from astrocytes accelerates $\mathrm{A} \beta$-induced neuronal toxicity. It is well recognised that the activation of astrocytes is accompanied by increased production of potentially neurotoxic factors, including cytokines, nitric oxide (NO) and reactive oxygen species, ${ }^{34}$ and $\mathrm{A} \beta$ can induce the production and secretion of $\mathrm{IL}-1 \beta, \mathrm{NO}$ and other inflammatory cytokines in cultured astrocytes and microglia. ${ }^{35}$ Here, we took an unbiased approach to show that $A \beta$ treatment significantly increases the secretion of several inflammatory cytokines and chemokines from primary astrocytes, and that minocycline-mediated protection from $\mathrm{A} \beta$-induced neurotoxicity was associated with the decreased secretion of inflammatory mediators from astrocytes. We found that the amounts of CINC2 $\alpha / \beta$, IFN- $\gamma$, IL- $1 \beta$, IL-1ra, IL-6, $\mathrm{IL}-13$, IL-17, IP-10 and MIG in culture medium were significantly increased by $\mathrm{A} \beta$, and these increases were significantly inhibited by the anti-inflammatory agent, minocycline. This suggests that these cytokines, in particular, may have an important role in the neurotoxic events downstream of $\mathrm{A} \beta$.

IL-17 belongs to the same cytokine subfamily as IL-6, acting to induce IL-6 expression in astrocytes. ${ }^{13}$ Thus, this finding is in agreement with those of Jana et al. ${ }^{11}$ who recently reported that astrocytes induce neuronal death by sphingomyelinasemediated induction of IL-1 $\beta$ and IL-6, together with TNF $\alpha$. Indeed, substantial evidence now indicates that IL-1 $\beta$ and IL-6/IL-17 have important roles in the development and/ or progression of $A D$, with these cytokines found in affected brain regions in association with plaques and tangles in $\mathrm{AD}$ brain, ${ }^{34}$ and transgenic mouse models of AD. ${ }^{4,36} \mathrm{We}$ also found that increased IP-10 secretion from astrocytes was associated with $\mathrm{A} \beta$-induced neurotoxicity. IP-10 is markedly increased in reactive astrocytes in $A D$ brains, and astrocytes positive for IP-10 are associated with senile plaques. ${ }^{37}$ To our knowledge, the findings presented here provide the first evidence for a direct effect of $A \beta$ on IP-10 production and thus may implicate IP-10 in AD pathogenesis.

In addition to $\mathrm{A} \beta$-induced neurotoxicity, we have examined the role of astrocytes in $\mathrm{A} \beta$-induced abnormal tau processing. We found that astrocytes augment $\mathrm{A} \beta$-induced caspase- 3 cleavage of tau, in keeping with our previous results. ${ }^{17}$ We also describe, for the first time, that astrocytes are required for $\mathrm{A} \beta$-induced tau phosphorylation, at least in this model system. Receptor binding of various cytokines and chemokines leads to the activation of protein kinase $C$, p38, ERK and JNK mitogen-activated protein kinases and PI3 kinase. ${ }^{13}$ Thus, it is possible that astrocyte-derived cytokines bind to neuronal plasma membrane receptors, inducing specific signalling pathways that result in the activation of tau kinases implicated in aberrant tau phosphorylation and cleavage in AD and the tauopathies. For example, binding of secreted IL-1 to neuronal IL-1 receptors leads to the activation of p38 MAPK and tau hyperphosphorylation in vivo. ${ }^{38}$ Activation of caspase-3 also occurs downstream of IL-1 receptors, ${ }^{39}$ which conceivably results in the C-terminal cleavage of tau to generate neurotoxic tau fragments. ${ }^{18}$ Our results indicate that it is predominantly the caspase-3-cleaved tau fragments that are more heavily phosphorylated following $\mathrm{A} \beta$ treatment of primary cortical cultures, and thus uncovering the mechanism by which astrocytes influence neuronal caspase-3 activity is likely to elucidate the mechanism by which $\mathrm{A} \beta$ increases aberrant tau processing, at least in this model system. The mechanism underlying the influence of astrocytes during $A \beta$-induced tau phosphorylation is currently under investigation in our laboratory.

It is interesting to note, however, that individual cytokines have been reported to have opposing effects on the development of tau and amyloid pathology in transgenic mouse models of AD. ${ }^{40}$ Indeed, we have shown previously that several of the cytokines identified here, including IL-17, as being associated with $\mathrm{A} \beta$-associated neurotoxicity, negatively correlated with the amount of tau phosphorylation in htau mice. ${ }^{6}$ Thus, further investigation is warranted into the roles of specific inflammatory cytokines in models of $A D$. Furthermore, it will be important to investigate the findings presented here in intact brain in which the potential influences of microglia-astrocyte signalling and complex neurotransmitter interactions can be investigated in an environment comprising approximately equivalent glia and neurons.

In summary, the results presented here indicate that astrocytes have an important role in mediating the effects of $\mathrm{A} \beta$ on cultured neurons. The influence of astrocytes in this system is closely related to the increased secretion of various inflammatory factors, including $\mathrm{IL}-1 \beta, \mathrm{IL}-17$ and IP-10. Although we cannot rule out the possibility that additional astrocyte-derived soluble factors may have a role in the effects on neurons reported here, these results provide further support for the investigation of anti-inflammatory strategies, or the specific inhibition of selected inflammatory cytokines, as potential therapies for the treatment of $A D$ and related neurodegenerative diseases. 


\section{Materials and Methods}

All materials were obtained from Sigma (Poole, Dorset, UK), unless otherwise stated.

Primary cell culture and cell treatments. Primary mixed cortical cultures were prepared from embryonic day 18 (E18) rat embryos and cultured as described previously. ${ }^{17}$ To establish neuronal cultures, the proliferation of glial cells in mixed cultures was prevented by the addition of $2 \mu \mathrm{M}$ cytosine arabinoside to three DIV cultures for $48 \mathrm{~h}$. Primary astrocytes were obtained from postnatal day 2 (P2) rats and cultured as described previously. ${ }^{41}$ At 2 days before treatment, astrocytes were re-plated into six-well dishes and subsequently maintained in serum-free medium. Cultures were treated with $10 \mu \mathrm{M}$ soluble oligomeric $\mathrm{A} \beta_{1-42}$ (California Peptide Co., Napa, CA, USA) or $20 \mu \mathrm{M}$ minocycline for $24 \mathrm{~h}$ before the addition of $\mathrm{A} \beta$ or vehicle $\left(\mathrm{H}_{2} \mathrm{O}\right)$. Soluble $A \beta_{1-42}$ was prepared according to the method described in Town et al., ${ }^{21}$ which we confirmed generates predominantly soluble oligomeric forms of $\mathrm{A} \beta$.

Toxicity assays. Cytotoxicity was evaluated by measuring LDH in culture medium using Cytotox 96 assay kits (Promega, Madison, WI, USA) according to the manufacturer's directions. After collection of medium, the remaining cells were lysed in $0.9 \%(w / v)$ Triton X-100, and LDH content in medium and lysed cells was measured to determine total LDH content. Optical density was measured at $492 \mathrm{~nm}$ (Wallac 1420 Victor3 plate reader, Perkin-Elmer, Waltham, MA, USA). LDH release from cells was calculated as a percentage of total LDH in each sample.

Dead cells in culture were identified by a live/dead fixable cell stain (Invitrogen, Paisley, UK) according to the manufacturer's instructions. Following uptake of the amine-reactive fluorescent dye provided, cells were washed, fixed and immunolabelled with an antibody against GFAP, as described below.

A $\beta_{1-42}$ ELISA. Semiquantitative ELISAs were performed as described previously, ${ }^{42}$ using the $6 \mathrm{E} 10$ antibody (Eurogentec, Southampton, UK) to detect $\mathrm{A} \beta_{1-42}$ in culture medium. $A \beta_{1-42}$ standards of known concentration were plated to generate a standard curve from which $A \beta$ content was measured in the medium of primary cortical cultures.

Immunocytochemistry. Cultured cells were fixed in $4 \%$ (w/v) paraformaldehyde in phosphate-buffered saline (PBS) for 5 min at $37^{\circ} \mathrm{C}$. Following fixation, cells were permeabilised $(0.3 \%$ (v/v) Triton X-100 in PBS) and blocked with $1 \%(\mathrm{w} / \mathrm{v})$ bovine serum albumin before incubation with monoclonal antibodies against GFAP (Dako, Ely, UK) or $\beta$ III-tubulin (Cell Signaling, Beverly, MA, USA). Cells were incubated with the appropriate species of secondary antibody and cell nuclei were stained with Hoescht $33342(5 \mu \mathrm{g} / \mathrm{ml}$ bisbenzimide in PBS). Neurons were examined using a Zeiss Axioskop microscope (Carl Zeiss Inc., Welwyn Garden City, UK). For cell counts, five separate non-overlapping images were taken using the $\times 20$ objective. Cell counts were made using the ImageJ software (National Institutes of Health, Bethesda, MD, USA).

Preparation of neuronal lysates. After treatments, the medium was removed and cells were washed in ice-cold PBS, followed by lysis in extra strong lysis buffer (100 mM Tris- $\mathrm{HCl}$ (pH 7.5), 0.5\% (w/v) sodium dodecyl sulphate (SDS), $0.5 \%$ (w/v) sodium deoxycholate, $1 \%(\mathrm{v} / \mathrm{v})$ Triton X-100, $75 \mathrm{mM}$ sodium chloride, $10 \mathrm{mM}$ ethylenediaminetetraacetic acid, $2 \mathrm{mM}$ sodium orthovanadate, $1.25 \mathrm{mM}$ sodium fluoride and protease inhibitor cocktail for mammalian tissues), and centrifugation at $16000 \times g_{\mathrm{av}}$ for $20 \mathrm{~min}$ at $4^{\circ} \mathrm{C}$. The protein concentration of supernatants was measured using a BCA protein assay kit (Pierce Endogen, Rockford, IL, USA) and samples were standardised to equal protein concentration before being analysed on SDS-PAGE.

SDS-PAGE and Immunoblotting. In total, 5-20 $\mu$ g protein was separated on 10 or $12 \%(\mathrm{w} / \mathrm{v})$ SDS-PAGE gels and electrophoretically transferred to nitrocellulose membrane. After blocking with $5 \%(\mathrm{w} / \mathrm{v})$ non-fat dried milk for $1 \mathrm{~h}$, membranes were probed with primary antibodies, followed by fluorophore-coupled secondary antibodies. Detected proteins were visualised and quantified, using an Odyssey infrared imaging system (Li-Cor Biosciences, Cambridge, UK). The following primary antibodies were used for western blotting. Total tau (rabbit polyclonal; Dako); tau-C3 (tau truncated at Asp421, mouse lgG1; Millipore, Billerica, MA, USA); tau (pSer396) (rabbit polyclonal; Invitrogen Biosource, Carlsbad, CA, USA); cleaved caspase-3 (Asp175, rabbit IgG; Cell Signaling); and $\beta$-actin (mouse
IgG1, clone AC-15; Abcam, Cambridge, UK). The following tau antibodies were kindly gifted by Peter Davies (Albert Einstein College of Medicine, Bronx, NY, USA): CP13 (phospho-Ser-202; mouse IgG1) and PHF-1 (phospho-Ser-396/404; mouse lgG1).

Cytokine antibody arrays. The medium from astrocyte-enriched cultures was collected and stored at $-20^{\circ} \mathrm{C}$ pending analysis of pro-inflammatory mediator content using Rat Proteome Profiler antibody arrays (R\&D Systems, Minneapolis, MN, USA). Before analysis, samples of medium were thawed on ice and centrifuged at $10000 \times g_{\mathrm{av}}$ for $1 \mathrm{~min}$ at $4^{\circ} \mathrm{C}$. Media from all experiments were processed in parallel to enable comparisons between experiments. Cytokine and chemokine content was measured according to the manufacturer's instructions. Briefly, array membranes were incubated in blocking buffer for $1 \mathrm{~h}$ at ambient temperature. Samples of medium were mixed with a cocktail of biotinylated detection antibodies before incubation at $4^{\circ} \mathrm{C}$ with the array membranes. Following washing, membranes were incubated with streptavidin-horseradish peroxidase for $30 \mathrm{~min}$ at ambient temperature. Immunoreactive species were visualised using enhanced chemoluminescence. Positive and negative controls included in each membrane allowed quantitative analysis by densitometry, with results expressed as percentage change from control cultures.

Statistics. Data were analysed using either Student's unpaired t-test or one-way analysis of variance (Graphpad Prism 5.0. Software, Graphpad Software Inc., La Jolla, CA, USA), followed by Bonferroni (for grouped) and Newman-Keuls (for all other analyses) post hoc tests. Differences were considered statistically significant when $P<0.05$.

\section{Conflict of Interest}

The authors declare no conflict of interest.

Acknowledgements. Work in the authors' laboratories is supported by funding from the Alzheimer's Society, Medical Research Council, the Henry Smith Charity and the Wellcome Trust.

1. McGeer EG, McGeer PL. Neuroinflammation in Alzheimer's disease and mild cognitive impairment: a field in its infancy. J Alzheimer's Dis 2010; 19: 355-361.

2. Cagnin A, Brooks DJ, Kennedy AM, Gunn RN, Myers R, Turkheimer FE et al. In-vivo measurement of activated microglia in dementia. Lancet 2001; 358: 461-467.

3. Parachikova A, Agadjanyan MG, Cribbs DH, Blurton-Jones M, Perreau V, Rogers J et al. Inflammatory changes parallel the early stages of Alzheimer disease. Neurobiol Aging 2007; 28: 1821-1833.

4. Schwab C, Klegeris A, McGeer PL. Inflammation in transgenic mouse models of neurodegenerative disorders. Biochim Biophys Acta 2010; 1802: 889-902.

5. Noble W, Hanger DP, Gallo JM. Transgenic mouse models of tauopathy in drug discovery. CNS Neurol Disord Drug Targets 2010; 9: 403-428.

6. Garwood C, Cooper JD, Hanger DP, Noble W. Anti-inflammatory effects of minocycline in a mouse model of tauopathy. Front Psych 2010; 1: 1-8.

7. Schindowski K, Bretteville A, Leroy K, Begard S, Brion JP, Hamdane M et al. Alzheimer's disease-like tau neuropathology leads to memory deficits and loss of functional synapses in a novel mutated tau transgenic mouse without any motor deficits. Am J Pathol 2006; 169: 599-616.

8. Parachikova A, Vasilevko V, Cribbs DH, LaFerla FM, Green KN. Reductions in amyloidbeta-derived neuroinflammation, with minocycline, restore cognition but do not significantly affect tau hyperphosphorylation. J Alzheimer's Dis 2010; 21: 527-542.

9. Simard AR, Soulet D, Gowing G, Julien JP, Rivest S. Bone marrow-derived microglia play a critical role in restricting senile plaque formation in Alzheimer's disease. Neuron 2006; 49: 489-502.

10. Hickman SE, Allison EK, El KJ. Microglial dysfunction and defective beta-amyloid clearance pathways in aging Alzheimer's disease mice. J Neurosci 2008; 28: 8354-8360.

11. Jana A, Pahan K. Fibrillar amyloid-beta-activated human astroglia kill primary human neurons via neutral sphingomyelinase: implications for Alzheimer's disease. J Neurosci 2010; 30: 12676-12689.

12. Whitney NP, Eidem TM, Peng H, Huang Y, Zheng JC. Inflammation mediates varying effects in neurogenesis: relevance to the pathogenesis of brain injury and neurodegenerative disorders. J Neurochem 2009; 108: 1343-1359.

13. Anisman $\mathrm{H}$. Cascading effects of stressors and inflammatory immune system activation: implications for major depressive disorder. J Psychiatry Neurosci 2009; 34: 4-20.

14. Van Eldik LJ, Thompson WL, Ralay RH, Behanna HA, Martin WD. Glia proinflammatory cytokine upregulation as a therapeutic target for neurodegenerative diseases: functionbased and target-based discovery approaches. Int Rev Neurobiol 2007; 82: 277-296. 
15. Mattson MP, Partin J, Begley JG. Amyloid beta-peptide induces apoptosis-related events in synapses and dendrites. Brain Res 1998; 807: 167-176.

16. Chung CW, Song YH, Kim IK, Yoon WJ, Ryu BR, Jo DG et al. Proapoptotic effects of tau cleavage product generated by caspase-3. Neurobiol Dis 2001; 8: 162-172.

17. Noble W, Garwood C, Stephenson J, Kinsey AM, Hanger DP, Anderton BH. Minocycline reduces the development of abnormal tau species in models of Alzheimer's disease. FASEB J 2009; 23: 739-750.

18. Gamblin TC, Chen F, Zambrano A, Abraha A, Lagalwar S, Guillozet AL et al. Caspase cleavage of tau: linking amyloid and neurofibrillary tangles in Alzheimer's disease. Proc Natl Acad Sci USA 2003; 100: 10032-10037.

19. Rissman RA, Poon WW, Blurton-Jones M, Oddo S, Torp R, Vitek MP et al. Caspasecleavage of tau is an early event in Alzheimer disease tangle pathology. J Clin Invest 2004 114: $121-130$.

20. Ballatore C, Lee VM, Trojanowski JQ. Tau-mediated neurodegeneration in Alzheimer's disease and related disorders. Nat Rev Neurosci 2007; 8: 663-672.

21. Town T, Zolton J, Shaffner R, Schnell B, Crescentini R, Wu Y et al. p35/Cdk5 pathway mediates soluble amyloid-beta peptide-induced tau phosphorylation in vitro. J Neurosci Res 2002; 69: 362-372.

22. Hanger DP, Anderton $\mathrm{BH}$, Noble W. Tau phosphorylation: the therapeutic challenge for neurodegenerative disease. Trends $\mathrm{Mol}$ Med 2009; 15: 112-119.

23. Nimmerjahn A. Astrocytes going live: advances and challenges. J Physiol 2009; 587 (Part 8): 1639-1647.

24. Hatten ME. Neuronal regulation of astroglial morphology and proliferation in vitro. J Cell Biol 1985; 100: 384-396

25. Escartin C, Bonvento G. Targeted activation of astrocytes: a potential neuroprotective strategy. Mol Neurobiol 2008; 38: 231-241.

26. Hu J, LaDu MJ, Van Eldik LJ. Apolipoprotein E attenuates beta-amyloid-induced astrocyte activation. J Neurochem 1998; 71: 1626-1634.

27. Chen Y, Vartiainen NE, Ying W, Chan PH, Koistinaho J, Swanson RA. Astrocytes protect neurons from nitric oxide toxicity by a glutathione-dependent mechanism. J Neurochem 2001; 77: 1601-1610.

28. Cribbs DH, Poon WW, Rissman RA, Blurton-Jones M. Caspase-mediated degeneration in Alzheimer's disease. Am J Pathol 2004; 165: 353-355.

29. Cho JH, Johnson GV. Glycogen synthase kinase 3 beta induces caspase-cleaved tau aggregation in situ. J Biol Chem 2004; 279: 54716-54723.

30. Hanger DP, Byers HL, Wray S, Leung KY, Saxton MJ, Seereeram A et al. Nove phosphorylation sites in tau from Alzheimer brain support a role for casein kinase 1 in disease pathogenesis. J Biol Chem 2007; 282: 23645-23654.
31. Paradisi S, Sacchetti B, Balduzzi M, Gaudi S, Malchiodi-Albedi F. Astrocyte modulation of in vitro beta-amyloid neurotoxicity. Glia 2004; 46: 252-260.

32. Olabarria M, Noristani HN, Verkhratsky A, Rodriguez JJ. Concomitant astroglial atrophy and astrogliosis in a triple transgenic animal model of Alzheimer's disease. Glia 2010; 58: $831-838$

33. Wallach D. Cell death induction by TNF: a matter of self control. Trends Biochem Sci 1997; 22: 107-109.

34. Wyss-Coray T. Inflammation in Alzheimer disease: driving force, bystander or beneficial response? Nat Med 2006; 12: 1005-1015.

35. Tuppo EE, Arias HR. The role of inflammation in Alzheimer's disease. Int J Biochem Cell Biol 2005; 37: 289-305

36. Mehlhorn G, Hollborn M, Schliebs R. Induction of cytokines in glial cells surrounding cortical beta-amyloid plaques in transgenic Tg2576 mice with Alzheimer pathology. Int J Dev Neurosci 2000; 18: 423-431.

37. Xia MQ, Bacskai BJ, Knowles RB, Qin SX, Hyman BT. Expression of the chemokine receptor $\mathrm{CXCR} 3$ on neurons and the elevated expression of its ligand IP-10 in reactive astrocytes: in vitro ERK1/2 activation and role in Alzheimer's disease. J Neuroimmunol 2000; 108: 227-235.

38. Bhaskar K, Konerth M, Kokiko-Cochran ON, Cardona A, Ransohoff RM, Lamb BT. Regulation of tau pathology by the microglial fractalkine receptor. Neuron 2010; 68: 19-31.

39. Lynch AM, Lynch MA. The age-related increase in IL-1 type I receptor in rat hippocampus is coupled with an increase in caspase-3 activation. Eur J Neurosci 2002; 15: 1779-1788.

40. Mastrangelo MA, Sudol KL, Narrow WC, Bowers WJ. Interferon-\{gamma\} differentially affects Alzheimer's disease pathologies and induces neurogenesis in triple transgenic-AD mice. Am J Pathol 2009; 175: 2076-2088.

41. Pinto SS, Gottried C, Mendez A, Goncalves D, Karl J, Goncalves CA et al. Immunocontent and secretion of $S 100 B$ in astrocyte cultures from different brain regions in relation to morphology. FEBS Lett 2000; 486: 203-207.

42. Kelleher I, Garwood C, Hanger DP, Anderton BH, Noble W. Kinase activities increase during the development of tauopathy in htau mice. J Neurochem 2007; 103: 2256-2267.

Cell Death and Disease is an open-access journal published by Nature Publishing Group. This work is licensed under the Creative Commons Attribution-Noncommercial-No Derivative Works 3.0 Unported License. To view a copy of this license, visit http://creativecommons.org/licenses/by-nc-nd/3.0/

\section{Supplementary Information accompanies the paper on Cell Death and Disease website (http://www.nature.com/cddis)}

